\title{
Effect of Statin Use on Liver Cancer Mortality Considering Hypercholesterolemia and Obesity in Patients with Non-Cirrhotic Chronic Hepatitis B
}

\author{
Gi-Ae Kim ${ }^{1}$, Jae-Jun Shim ${ }^{1}$, Ji Sung Lee ${ }^{2,3}$, Byung-Ho Kim¹ ${ }^{1}$ Jung Wook Kim¹, \\ Chi Hyuk $\mathrm{Oh}^{1}$, Chang-Mo $\mathrm{Oh}^{4}$, In-Hwan $\mathrm{Oh}^{4}$, and So-Youn Park ${ }^{5}$ \\ ${ }^{1}$ Department of Internal Medicine, School of Medicine, Kyung Hee University, Seoul; \\ ${ }^{2}$ Department of Clinical Epidemiology and Biostatistics, Asan Medical Center, University of Ulsan College of Medicine, Seoul; \\ ${ }^{3}$ Clinical Research Center, Asan Institute for Life Sciences, Asan Medical Center, University of Ulsan College of Medicine, Seoul; \\ ${ }^{4}$ Department of Preventive Medicine, School of Medicine, Kyung Hee University, Seoul; \\ ${ }^{5}$ Department of Medical Education and Humanities, School of Medicine, Kyung Hee University, Seoul, Korea.
}

\begin{abstract}
Little is known about the benefits of statin use on liver cancer mortality among patients with chronic hepatitis B (CHB) considering hypercholesterolemia and obesity. A nationwide retrospective cohort study was conducted using data from a Health Examination Cohort of the National Health Insurance Service of Korea. Data on CHB patients with no other concurrent liver disease were acquired, and statin use was defined as a cumulative daily dose $\geq 28$. A 3-year landmark analysis was performed to avoid immortal time bias. Patients who started statin therapy within the landmark date were considered statin users. A Cox regression analysis was applied to assess associations between statin use and liver cancer mortality considering hypercholesterolemia and obesity. Among 13063 patients, 193 (1.5\%) died of liver cancer during the mean follow-up period of 10.6 years. After adjusting for demographic and metabolic factors, statin use [hazard ratio (HR), 0.17; 95\% confidence interval (CI), 0.04-0.70] and hypercholesterolemia (HR, 0.46 ; $95 \%$ CI, $0.24-0.88$ for total cholesterol $\geq 240 \mathrm{mg} / \mathrm{dL}$ ) were associated with a decreased risk of liver cancer mortality, whereas body mass index (BMI) $\geq 30 \mathrm{~kg} / \mathrm{m}^{2}$ was associated with an increased risk of liver cancer mortality (HR, 2.46; 95\% CI, 1.20-5.06). This study showed that statin use was associated with decreased liver cancer mortality when adjusting for cholesterol levels and BMI. This study found that hypercholesterolemia was independently associated with decreased liver cancer mortality regardless of statin use.
\end{abstract}

Key Words: Chronic hepatitis B, hepatocellular carcinoma, mortality, cholesterol

Hepatitis B virus (HBV) infection is a major global health burden. ${ }^{1}$ An estimated 291 million people are chronically infected with $\mathrm{HBV}$, a leading cause of cancer-related mortality worldwide. ${ }^{2}$

Received: July 3, 2019 Revised: September 17, 2019

Accepted: October 16, 2019

Corresponding author: Jae-Jun Shim, MD, PhD, Department of Internal Medicine, School of Medicine, Kyung Hee University, 26 Kyungheedae-ro, Dongdaemungu, Seoul 02447, Korea.

Tel: 82-2-958-8155, Fax: 82-2-968-1848, E-mail: joyshim@khu.ac.kr

-The authors have no potential conflicts of interest to disclose.

(C) Copyright: Yonsei University College of Medicine 2019

This is an Open Access article distributed under the terms of the Creative Commons Attribution Non-Commercial License (https://creativecommons.org/licenses/ by-nc/4.0) which permits unrestricted non-commercial use, distribution, and reproduction in any medium, provided the original work is properly cited.
Statins, also known as 3-hydroxy-3-methylglutaryl CoA reductase inhibitors, are widely used for treating dyslipidemia and preventing cardiovascular disease. ${ }^{3}$ Studies have reported a decreased risk of cancers after the use of statins, including breast cancer, colon cancer, and prostate cancer. ${ }^{4,5}$ The chemopreventive effects of statins may be related to growth and apoptosis effects on cancer cells. ${ }^{6}$ The beneficial effect of statin use on the development of hepatocellular carcinoma (HCC) in patients with chronic HBV infection, HCV infection, or cirrhosis has been reported. ${ }^{7-10}$ In addition, a previous study reported an association between statin use and a reduced risk of liver cancer and death among cirrhosis patients with a focus on hypercholesterolemia. ${ }^{11}$ However, limited data are available on whether the same beneficial effects of statin use can be ex- 
pected in patients with chronic hepatitis $\mathrm{B}$ (CHB) without cirrhosis when hypercholesterolemia or obesity are considered as confounding factors. Therefore, the present study sought to investigate the effect of statin use on liver cancer mortality in non-cirrhotic patients with CHB taking hypercholesterolemia and obesity into account.

A nationwide retrospective cohort study was conducted using data from a Health Examination Cohort of the National Health Insurance Service (NHIS) of Korea. The data collection method was the same as that in a previous study. ${ }^{12} \mathrm{~A}$ representative sample cohort of 514866 individuals was randomly selected from 5.15 million Koreans aged 40-79 years who underwent health examinations between January 1, 2002 and December 31, 2003. Patients with CHB ( $\mathrm{n}=22987)$ were selected from the database using the disease codes B18.0 or B18.1. Statin use was defined as a cumulative defined daily dose (cDDD) $\geq 28$ between 2003 and 2005. ${ }^{7}$ In order to avoid immortal time bias, we utilized the landmark analysis method. For patients who started statin therapy within this 3-year landmark date, the patients were designated into statin users. Both statin users and non-users were followed up from the landmark date to 2013. The primary outcome was liver cancer-related death and the disease code used was C22 (liver cancers). This study was approved by the Institutional Review Board of Kyung Hee University (KHSIRB-16-048).

The exclusion criteria were as follows: history of alcoholic liver disease or chronic hepatitis C (B 18.2) ( $\mathrm{n}=5388)$; liver cirrhosis (K74), liver cancer (C22), or other chronic liver diseases (B18.8, B18.9) at baseline ( $\mathrm{n}=3890)$; patients who had been treated with statins before the health examination $(n=127)$; patients who were treated with statins CDDD $\geq 28$ between January 2002 and December 2002 ( $\mathrm{n}=303)$; patients on other dyslipidemic agents before the health examination $(\mathrm{n}=10)$; and patients on other dyslipidemic agents between January 2002 and December 2002 ( $\mathrm{n}=37)$. Also, any patients who died during the statin exposure period were excluded $(n=169)$. A total of 13063 patients with CHB were enrolled. The baseline characteristics and laboratory test results of the study population were collected from the date closest to the date of the CHB diagnosis within 2 years. In cases wherein no data were available, we collected data within 1 year after the CHB diagnosis.

The characteristics of statin users were compared with those of non-users. Categorical and continuous variables were compared using the chi-square and t-test, respectively. Cumulative rates of liver cancer mortality were estimated using the Kaplan-Meier method and log lank test. Univariate and multivariable Cox proportional hazards regression analysis were performed to assess hazard ratios (HRs) of liver cancer mortality. Age, sex, body mass index (BMI), serum alanine aminotransferase (ALT) levels, $\gamma$-glutamyl transferase (GGT) levels, total cholesterol levels, fasting blood glucose levels, hypertension, family history of liver disease, smoking status, alcohol consumption, and statin use were included in multivariable analyses. All analyses were performed using SAS version 9.4 (SAS Institute; Cary, NC, USA). Two-sided $p$ values $<0.05$ were considered to indicate statistical significance.

The baseline characteristics of the study population are shown in Table 1. Of the 13063 patients, 980 (7.5\%) were statin users and 12083 (92.5\%) were statin-naïve. Statin users were older (mean age, 55 vs. 52 years, $p<0.001$ ), had higher BMI ( 24.9 vs. $23.9, p<0.001$ ), had higher levels of ALT ( 25 vs. $23 \mathrm{U} / \mathrm{L}$, $p=0.009$ ) and GGT ( 26 vs. $22 \mathrm{U} / \mathrm{L}, p<0.001$ ), and had a higher proportion of hypertension ( $43.5 \%$ vs. $30.2 \%, p<0.001)$, compared with non-users. The number of patients having total cholesterol levels $<200 \mathrm{mg} / \mathrm{dL}, 200-239 \mathrm{mg} / \mathrm{dL}$, and $\geq 240 \mathrm{mg} /$ dL were 218 (22.2\%), 374 (38.2\%), and 388 (39.6\%), respectively, among statin users and 7162 (59.4\%), 3765 (31.2\%), and 1139 (9.4\%), respectively, among non-users.

During the mean follow-up period of 10.6 years (standard deviation, 1.2 years), 193 patients (1.5\%) died of liver cancer. The annual liver cancer mortality was significantly lower among statin users [0.29 per 1000 person-year (PY)] than nonusers (1.48 per $1000 \mathrm{PY}$ ). The statin users had a significantly lower liver cancer mortality in univariate analysis [HR, 0.19; $95 \%$ confidence interval (CI), 0.06-0.61; Fig. 1, Table 2] and in age- and sex-adjusted analysis (HR, 0.20; 95\% CI, 0.06-0.62). Hypercholesterolemia was associated with a decreased risk of liver cancer mortality in univariate analysis (HR, 0.36 ; 95\% CI, $0.20-0.67$ for total cholesterol $\geq 240 \mathrm{mg} / \mathrm{dL}$ ) and in age- and sex-adjusted analysis (HR, 0.37 ; $95 \%$ CI, $0.20-0.69$ for total cholesterol $\geq 240 \mathrm{mg} / \mathrm{dL}$ ). High BMI ( $\geq 30 \mathrm{~kg} / \mathrm{m}^{2} ; \mathrm{HR}, 2.10 ; 95 \%$ CI, 1.04-4.21) was also associated with an increased risk of liver cancer mortality in the age- and sex-adjusted analysis.

Multivariable analysis after adjusting for demographic and metabolic factors showed consistent results. Statin use (HR, 0.17; 95\% CI, 0.04-0.70) and hypercholesterolemia (HR, 0.46; $95 \% \mathrm{CI}, 0.24-0.88$ for total cholesterol $\geq 240 \mathrm{mg} / \mathrm{dL}$ ) were associated with a decreased risk of liver cancer mortality (Table 2). High BMI ( $\left.\geq 30 \mathrm{~kg} / \mathrm{m}^{2} ; \mathrm{HR}, 2.46 ; 95 \% \mathrm{CI}, 1.20-5.06\right)$ was associated with an increased risk of liver cancer mortality.

The effect of statin use on liver cancer mortality among non-cirrhotic patients with CHB was investigated in this study, with a consideration paid to hypercholesterolemia and obesity. The results showed that statin use was associated with decreased liver cancer mortality when adjusting for cholesterol levels and BMI. The study also found that hypercholesterolemia was independently associated with decreased liver cancer mortality regardless of statin use.

A chemopreventive effect for statins has been reported in several studies. The anti-inflammatory, anti-angiogenic, and anti-tumor effects of statins can be related to a reduced risk of HCC development, and a lowered risk of HCC with statins has been reported worldwide. ${ }^{6}$ A meta-analysis of 10 studies showed statin use was associated with a $37 \%$ reduced risk of HCC. ${ }^{13}$ In addition, a recent study of patients with $\mathrm{CHB}$ showed that statin use can achieve a $33-44 \%$ risk reduction in HCC 
Table 1. Baseline Characteristics of the Study Patients

\begin{tabular}{|c|c|c|c|c|}
\hline Characteristics & Total (n=13063) & Statin user (n=980) & Statin non-user ( $\mathrm{n}=12083$ ) & $p$ value \\
\hline Age (yr) (mean \pm SD) & $52.1 \pm 9.0$ & $55.4 \pm 8.5$ & $51.8 \pm 9.0$ & $<0.001$ \\
\hline Male & $6658(51.0)$ & $403(41.1)$ & $6255(51.8)$ & $<0.001$ \\
\hline $\mathrm{BMI}\left(\mathrm{kg} / \mathrm{m}^{2}\right)($ mean $\pm \mathrm{SD})$ & $24.0 \pm 2.9$ & $24.9 \pm 3.0$ & $23.9 \pm 2.9$ & $<0.001$ \\
\hline$<18.5$ & $263(2.0)$ & $13(1.3)$ & $250(2.1)$ & $<0.001$ \\
\hline $18.5-22.9$ & 4606 (35.3) & $241(24.6)$ & $4365(36.2)$ & \\
\hline $23-24.9$ & 3564 (27.3) & $264(27.0)$ & $3300(27.3)$ & \\
\hline $25-29.9$ & $4280(32.8)$ & $411(42.0)$ & $3869(32.0)$ & \\
\hline$\geq 30$ & $340(2.6)$ & $50(5.1)$ & $290(2.4)$ & \\
\hline ALT (U/L) [median (IOR)] & $23(17-35)$ & $25(18-36)$ & $23(17-35)$ & 0.009 \\
\hline GGT (U/L) [median (IOR)] & $23(15-39)$ & $26(17-44)$ & $22(15-39)$ & $<0.001$ \\
\hline Total cholesterol (mg/dL) & & & & $<0.001$ \\
\hline$<200$ & $7380(56.6)$ & $218(22.2)$ & $7162(59.4)$ & \\
\hline $200-239$ & 4139 (31.7) & $374(38.2)$ & $3765(31.2)$ & \\
\hline$\geq 240$ & $1527(11.7)$ & $388(39.6)$ & $1139(9.4)$ & \\
\hline Fasting blood glucose $\geq 126 \mathrm{mg} / \mathrm{dL}$ & $975(7.5)$ & $140(14.3)$ & $835(6.9)$ & $<0.001$ \\
\hline Hypertension & 4078 (31.2) & $426(43.5)$ & $3652(30.2)$ & $<0.001$ \\
\hline Family history of liver disease & 959 (8.2) & $55(6.2)$ & $904(8.4)$ & $<0.001$ \\
\hline Smoking & & & & 0.008 \\
\hline Non-smoker & $8923(71.2)$ & $715(75.7)$ & 8208 (70.9) & \\
\hline Past smoker & $1104(8.8)$ & $70(7.4)$ & $1034(8.9)$ & \\
\hline Current smoker & 2498 (19.9) & $160(16.9)$ & $2338(20.2)$ & \\
\hline Alcohol consumption (g/day) & & & & $<0.001$ \\
\hline Non-drinker & $7894(61.7)$ & $651(68.0)$ & $7243(61.2)$ & \\
\hline$<50$ & $4323(33.8)$ & $277(28.9)$ & 4046 (34.2) & \\
\hline$\geq 50$ & $574(4.5)$ & $29(3.0)$ & $545(4.6)$ & \\
\hline Liver cancer mortality & $193(1.5)$ & $3(0.3)$ & $190(1.6)$ & $<0.001$ \\
\hline
\end{tabular}

BMI, body mass index; ALT, alanine aminotransferase; IQR, interquartile range; GGT, gamma-glutamyl transferase.

Data are presented as mean \pm standard deviation (SD) or number (\%) unless otherwise indicated.

Information regarding BMI, total cholesterol, family history of liver disease, smoking status, and alcohol consumption was obtained for 13053 patients (99.9\%), 13046 patients (99.9\%), 11695 (89.5\%), 12525 patients (95.9\%), and 12791 patients (97.9\%), respectively.

development. ${ }^{14}$ With the relatively short median follow-up of 4.6 years, the study was not able to exhibit a mortality benefit for statin use. With a longer follow-up of about 10 years, our study was able to show that statin use contributes to decreased liver cancer mortality. These findings may serve to justify the use of statins for chemoprevention in patients with CHB.

This study also found that hypercholesterolemia is associated with a mortality benefit regardless of statin use. This is in line with a previous study that found both statin use and hypercholesterolemia were associated with improved survival in patients with cirrhosis. ${ }^{11}$ With serum cholesterol levels as a surrogate marker of hepatic synthetic function, high cholesterol levels may suggest better survival of liver-related disease. ${ }^{11}$ The better survival shown among statin users may have been from hypercholesterolemia and independent of any pharmacologic effect of statins. This study found that both statin use and hypercholesterolemia were independent factors associated with decreased liver cancer mortality. Thus, attention should be paid to both cholesterol levels and statin use when treating patients with CHB.
The association between BMI $\geq 30 \mathrm{~kg} / \mathrm{m}^{2}$ and increased liver cancer mortality documented in this study was consistent with a previous study. ${ }^{15}$ A study conducted in Korea found a J-shaped pattern between BMI and death and showed the risk of death from cancer increased substantially among subjects with a BMI above $30.0 \mathrm{~kg} / \mathrm{m}^{2} .{ }^{15}$ Other studies have also reported that obesity was associated with a higher risk of liver cancer development both in the general population and in patients with CHB..$^{16,17}$

This study has some limitations. First, being a study based on observational data, this study may not be free of bias and confounding. As the NHIS of Korea does not incorporate serum HBV DNA levels or detailed data on liver cirrhosis status, neither was available for this study. In addition, the information on liver cancer incidence was not available in the given dataset. Second, although the impact of antiviral treatment, such as nucleos(t)ide analogues, on liver cancer mortality is significant, this study was not able to consider it, as data on the status of antiviral treatment in the study patients was not available. Third, cardiovascular mortality, which may have affected liver cancer mortality, was not taken into account as a com- 


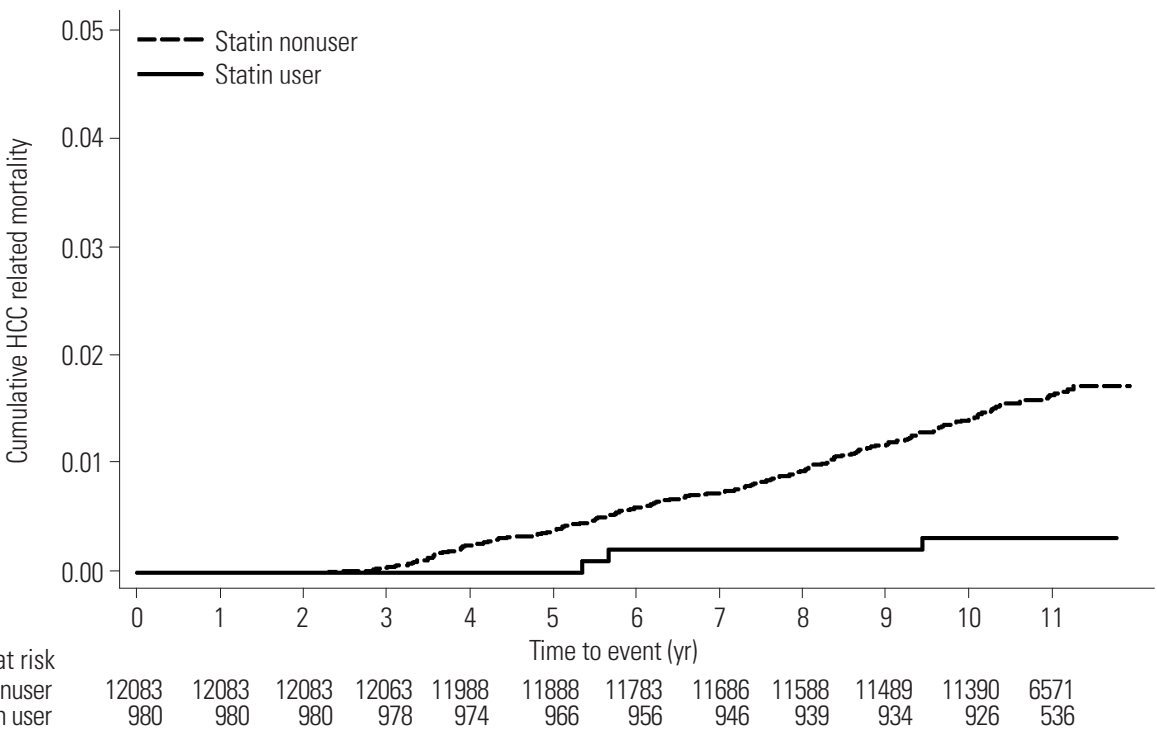

A

Number at risk Statin nonuse

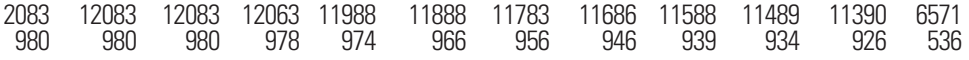

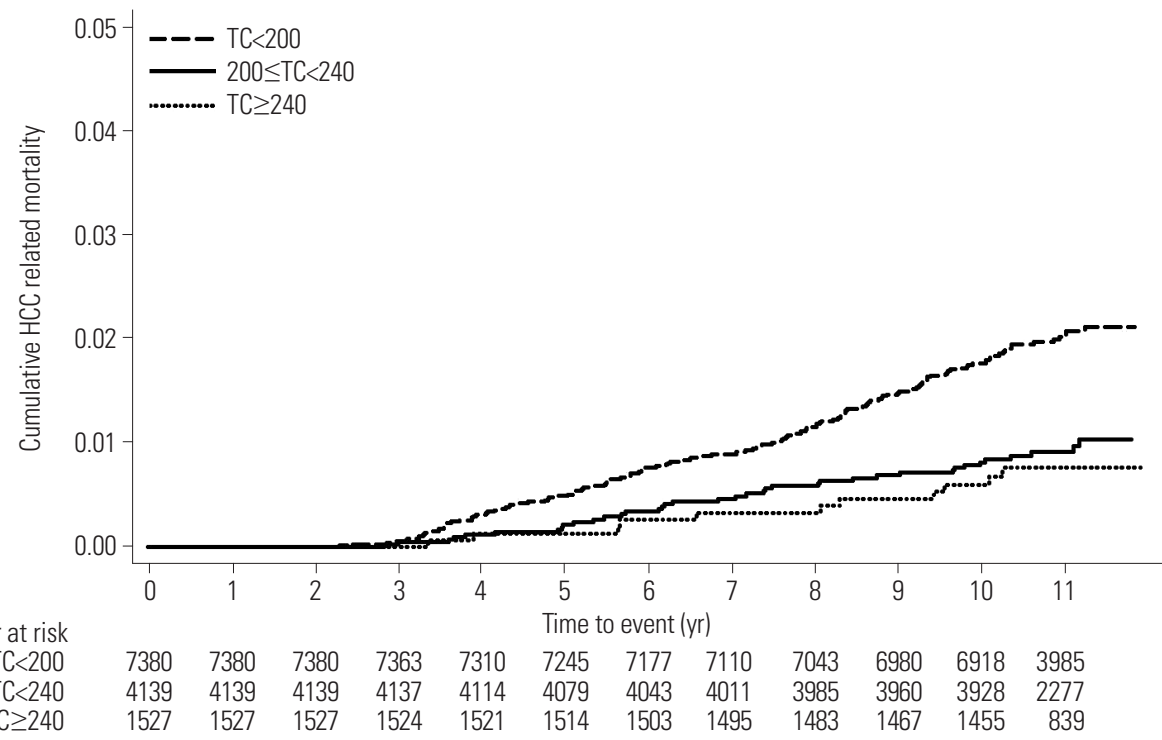

B

Number at risk
$T C<200$
$200 \leq T C<240$
$T C \geq 240$

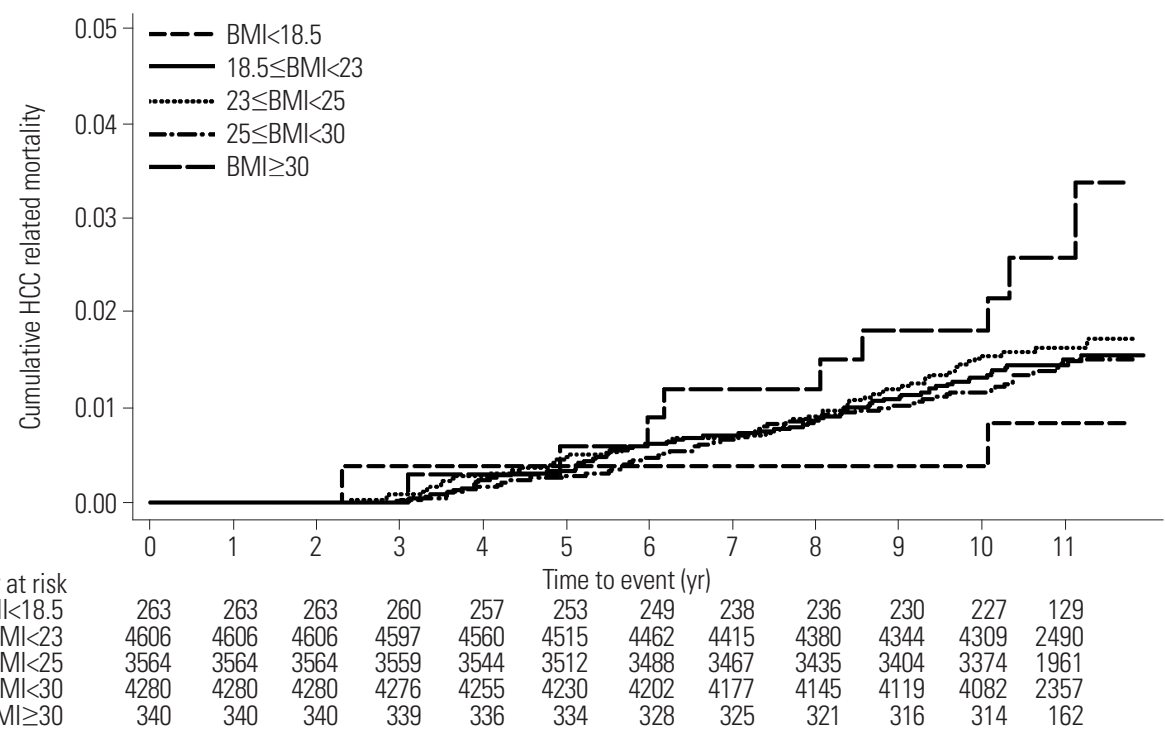

Fig. 1. Cumulative liver cancer mortality according to (A) statin use, (B) total cholesterol (TC), and (C) body mass index (BMI). HCC, hepatocellular carcinoma. 
Table 2. Liver Cancer Mortality in Unadjusted and Adjusted Analyses

\begin{tabular}{|c|c|c|c|c|}
\hline & $\begin{array}{l}\text { Mortality rate per } 1000 \text { PY } \\
\qquad(95 \% \mathrm{CI})\end{array}$ & $\begin{array}{l}\text { Univariate analysis } \\
\text { [HR }(95 \% \mathrm{CI})]\end{array}$ & $\begin{array}{l}\text { Age- and sex-adjusted analysis } \\
\text { [HR (95\% CI)] }\end{array}$ & $\begin{array}{c}\text { Multivariable analysis } \\
\text { [HR }(95 \% \mathrm{CI})]\end{array}$ \\
\hline \multicolumn{5}{|l|}{ Statin } \\
\hline No & $1.48(1.28-1.71)$ & Reference & Reference & Reference \\
\hline Yes & $0.29(0.09-0.89)$ & $0.19(0.06-0.61)$ & $0.20(0.06-0.62)$ & $0.17(0.04-0.70)$ \\
\hline \multicolumn{5}{|l|}{ BMI $\left(\mathrm{kg} / \mathrm{m}^{2}\right)$} \\
\hline$<18.5$ & $0.74(0.19-2.97)$ & $0.56(0.14-2.27)$ & $0.50(0.12-2.05)$ & $0.28(0.04-2.02)$ \\
\hline $18.5-22.9$ & $1.35(1.06-1.72)$ & Reference & Reference & Reference \\
\hline $23-24.9$ & $1.50(1.16-1.95)$ & $1.11(0.78-1.58)$ & $1.02(0.71-1.45)$ & $1.06(0.71-1.59)$ \\
\hline $25-29.9$ & $1.29(1.00-1.67)$ & $0.95(0.67-1.35)$ & $0.90(0.63-1.28)$ & $0.96(0.64-1.42)$ \\
\hline$\geq 30$ & $2.53(1.32-4.86)$ & $1.88(0.94-3.77)$ & $2.10(1.04-4.21)$ & $2.46(1.20-5.06)$ \\
\hline \multicolumn{5}{|c|}{ Total cholesterol (mg/dL) } \\
\hline$<200$ & $1.84(1.56-2.17)$ & Reference & Reference & Reference \\
\hline $200-239$ & $0.86(0.63-1.18)$ & $0.47(0.33-0.67)$ & $0.48(0.34-0.69)$ & $0.50(0.34-0.74)$ \\
\hline$\geq 240$ & $0.67(0.37-1.22)$ & $0.36(0.20-0.67)$ & $0.37(0.20-0.69)$ & $0.46(0.24-0.88)$ \\
\hline
\end{tabular}

$\mathrm{PY}$, person-years; $\mathrm{HR}$, hazard ratio; $\mathrm{Cl}$, confidence interval; $\mathrm{BMI}$, body mass index.

Total number of patients: 13063, Number of events: 193. Multivariable analysis was adjusted for age, sex, BMI, ALT, GGT, total cholesterol, fasting blood glucose, hypertension, family history of liver disease, smoking status, alcohol consumption, and statin use.

peting risk. Moreover, this study was not able to include multiple co-morbidities that might affect the prognosis of patients. Lastly, the study population consisted exclusively of Koreans, which limits the generalizability of the findings. All Koreans are infected by genotype $\mathrm{C} \mathrm{HBV},{ }^{18}$ which is mostly acquired through vertical transmission and is associated with a high risk of disease progression.

In this nationwide retrospective cohort of non-cirrhotic patients with $\mathrm{CHB}$, we found that hypercholesterolemia and $\mathrm{BMI} \geq 30 \mathrm{~kg} / \mathrm{m}^{2}$ were associated with liver cancer mortality. Moreover, statin use was found to be an independent predictor of decreased liver cancer mortality, regardless of cholesterol levels and BMI.

\section{AUTHOR CONTRIBUTIONS}

Conceptualization: Jae-Jun Shim. Data curation: Ji Sung Lee, ChangMo Oh, and So-Youn Park. Formal analysis: Jae-Jun Shim, Ji Sung Lee, and In-Hwan Oh. Funding acquisition: Jae-Jun Shim. Investigation: Gi-Ae Kim, Byung-Ho Kim, Jung Wook Kim, and Chi Hyuk Oh. Methodology: Jae-Jun Shim, Ji Sung Lee, and In-Hwan Oh. Project administration: Jae-Jun Shim, Jung Wook Kim, and Chi Hyuk Oh. Resources: Jae-Jun Shim. Software: Gi-Ae Kim. Supervision: Jae-Jun Shim and Byung-Ho Kim. Validation: Chang-Mo Oh, In-Hwan Oh, and So-Youn Park. Visualization: Gi-Ae Kim and Jae-Jun Shim. Writing-original draft: Gi-Ae Kim and Jae-Jun Shim. Writing_review \& editing: Gi-Ae Kim, Jae-Jun Shim, and Ji Sung Lee.

\section{ORCID iDs}

Gi-Ae Kim Jae-Jun Shim Ji Sung Lee Byung-Ho Kim https://orcid.org/0000-0002-5002-0822 https://orcid.org/0000-0003-2497-8663 https://orcid.org/0000-0001-8194-3462 https://orcid.org/0000-0002-1347-3523
Jung Wook Kim Chi Hyuk Oh

Chang-Mo Oh

In-Hwan Oh

So-Youn Park

https://orcid.org/0000-0002-5383-7934 https://orcid.org/0000-0002-4382-5876 https://orcid.org/0000-0002-5709-9350 https://orcid.org/0000-0002-5450-9887 https://orcid.org/0000-0003-0553-5381

\section{REFERENCES}

1. Stanaway JD, Flaxman AD, Naghavi M, Fitzmaurice C, Vos T, Abubakar I, et al. The global burden of viral hepatitis from 1990 to 2013: findings from the Global Burden of Disease Study 2013. Lancet 2016;388:1081-8.

2. Polaris Observatory Collaborators. Global prevalence, treatment, and prevention of hepatitis B virus infection in 2016: a modelling study. Lancet Gastroenterol Hepatol 2018;3:383-403.

3. Cholesterol Treatment Trialists' (CTT) Collaboration, Baigent C, Blackwell L, Emberson J, Holland LE, Reith C, et al. Efficacy and safety of more intensive lowering of LDL cholesterol: a metaanalysis of data from 170,000 participants in 26 randomised trials. Lancet 2010;376:1670-81.

4. Demierre MF, Higgins PD, Gruber SB, Hawk E, Lippman SM. Statins and cancer prevention. Nat Rev Cancer 2005;5:930-42.

5. Moon DC, Lee HS, Lee YI, Chung MJ, Park JY, Park SW, et al. Concomitant statin use has a favorable effect on gemcitabine-erlotinib combination chemotherapy for advanced pancreatic cancer. Yonsei Med J 2016;57:1124-30.

6. Lonardo A, Loria P. Potential for statins in the chemoprevention and management of hepatocellular carcinoma. J Gastroenterol Hepatol 2012;27:1654-64.

7. Tsan YT, Lee CH, Wang JD, Chen PC. Statins and the risk of hepatocellular carcinoma in patients with hepatitis B virus infection. J Clin Oncol 2012;30:623-30.

8. Tsan YT, Lee CH, Ho WC, Lin MH, Wang JD, Chen PC. Statins and the risk of hepatocellular carcinoma in patients with hepatitis $\mathrm{C}$ virus infection. J Clin Oncol 2013;31:1514-21.

9. Huang YW, Lee CL, Yang SS, Fu SC, Chen YY, Wang TC, et al. Statins reduce the risk of cirrhosis and its decompensation in chronic hepatitis B patients: a nationwide cohort study. Am J Gas- 
troenterol 2016;111:976-85.

10. Kim G, Jang SY, Nam CM, Kang ES. Statin use and the risk of hepatocellular carcinoma in patients at high risk: a nationwide nested case-control study. J Hepatol 2018;68:476-84.

11. Kaplan DE, Serper MA, Mehta R, Fox R, John B, Aytaman A, et al. Effects of hypercholesterolemia and statin exposure on survival in a large national cohort of patients with cirrhosis. Gastroenterology 2019;156:1693-706.

12. Shim JJ, Kim JW, Oh CH, Lee YR, Lee JS, Park SY, et al. Serum alanine aminotransferase level and liver-related mortality in patients with chronic hepatitis B: a large national cohort study. Liver Int 2018;38:1751-9.

13. Singh S, Singh PP, Singh AG, Murad MH, Sanchez W. Statins are associated with a reduced risk of hepatocellular cancer: a systematic review and meta-analysis. Gastroenterology 2013;144:323-32.

14. Hsiang JC, Wong GL, Tse YK, Wong VW, Yip TC, Chan HL. Statin and the risk of hepatocellular carcinoma and death in a hospital- based hepatitis B-infected population: a propensity score landmark analysis. J Hepatol 2015;63:1190-7.

15. Jee SH, Sull JW, Park J, Lee SY, Ohrr H, Guallar E, et al. Body-mass index and mortality in Korean men and women. N Engl J Med 2006;355:779-87.

16. Chen Y, Wang X, Wang J, Yan Z, Luo J. Excess body weight and the risk of primary liver cancer: an updated meta-analysis of prospective studies. Eur J Cancer 2012;48:2137-45.

17. Kim K, Choi S, Park SM. Association of high body mass index and hepatocellular carcinoma in patients with chronic hepatitis B virus infection: a Korean population-based cohort study. JAMA Oncol 2018;4:737-9.

18. Kim H, Jee YM, Song BC, Shin JW, Yang SH, Mun HS, et al. Molecular epidemiology of hepatitis B virus (HBV) genotypes and serotypes in patients with chronic HBV infection in Korea. Intervirology 2007;50:52-7. 